\title{
How do ALOHA and Listen Before Talk Coexist in LoRaWAN?
}

\author{
Jorge Ortín \\ Centro Universitario de la Defensa, \\ 50090 Zaragoza, Spain \\ jortin@unizar.es
}

\author{
Matteo Cesana, Alessandro Redondi \\ Dipartimento di Elettronica, Informazione e Bioingegneria, \\ Politecnico di Milano, \\ Milano, Italy \\ \{matteo.cesana, alessandroenrico.redondi\}@polimi.it
}

\begin{abstract}
In this work we address the analysis of a LoRaWAN network where some devices access the channel according to the standard-compliant ALOHA protocol, while other devices transmit according to a Listen Before Talk paradigm based on the CSMA/CA mechanism. To analyze this scenario, we propose a mathematical model to obtain the Data Extraction Rate both for CSMA/CA and ALOHA devices, as well as the average delay experienced by messages transmitted by CSMA/CA devices. Simulation results show the accuracy of our model, as well as the benefits of introducing CSMA/CA devices into the network, even when not all the devices implement this mechanism and must coexist with ALOHA devices.
\end{abstract}

\section{INTRODUCTION}

The Internet of Things (IoT) vision is based on the idea of bringing (Internet) connectivity to unmanned devices immersed in the environment which can then enable a vast range of vertical applications including smart cities, environmental monitoring, e-health and surveillance. Most of these application scenarios are characterized by highly dense networks of field devices which intermittently generate small amount of data; such new traffic/network paradigm is often referred to as Machine-Type Communications opposed to the classical human-to-human services offered by mobile cellular networks [1].

New communication standards and solutions are being rolled out to cope with such new traffic paradigm. Mobile cellular operators are working to evolve "legacy" mobile cellular standards towards MTC-compliant solutions including LTE-M, EC-GSM, NB-IoT and 5G; at the same time, IoT-specialized network operators are gaining momentum by selling IoT connectivity through long-range, low-power wireless technologies like SigFox, LoRaWAN [2], Weightless and Ingenu. Such low-power wireless technologies share the same proposition value of low energy consumption and Total Cost of Ownership (TCO), global reach and plug-and-play connectivity.

We focus here on LoRaWAN, which is characterized by an association-less star-of-stars topology in which end devices use single-hop spread spectrum wireless transmission to reach one or multiple gateways that relay messages towards a central network server in the backend. The performance of LoRaWAN networks in terms of coverage, end-to-end latency and Data Extraction Rate (DER) depend jointly on the network layout (number/position of the gateways), the configuration of the physical layer parameters (spreading factor, protection coding rate, channel bandwidth, emitted power) and the efficiency of the Medium Access Control (MAC) scheme.

Broadly speaking, the related work on the performance evaluation of LoRaWAN networks is either based on coverage assessment through experimental tests with commercial LoRa transceivers [3] - [5] or based on system level simulation [6] - [8]. At the moment of writing, there are few works proposing theoretical models to capture LoRaWAN performance. Delobel et al. propose in [9] a Markovian analysis to assess the performance of Class B devices, whereas Georgious et al. propose in [10] a theoretical framework based on stochastic geometry to derive the uplink outage probability in LoRaWAN. Along the same lines, Zucchetto et al. analyze in [11] the performance of random access solutions for long-range technologies.

The MAC scheme adopted by LoRaWAN is based on simple ALOHA, which is proved to be a major performance bottleneck as the network size grows. Stimulated by this fact, we provide two main novel contributions with respect to the reference literature: (i) we explore the possibility of using Listen Before Talk (LBT) approaches together with ALOHA at the MAC layer of LoRaWAN networks; (ii) we propose a comprehensive theoretical framework based on Markovian analysis to assess the performance of Class A devices in such scenarios. In detail, we consider network scenarios heterogeneous from the MAC point of view with end devices running the standard ALOHA-based access scheme and other end devices operating according to LBT approaches. The proposed framework is used to derive the DER and the transmission delay in such mixed environment and is validated against simulation.

The manuscript is organized as follows: Section 
II gives a brief overview of LoRaWAN; Section III describes the proposed modeling, whereas Section IV reports and comments on the performance evaluation campaign. Concluding remarks are given in Section V.

\section{LORAWAN OVERVIEW}

LoRaWAN operates in the unlicensed radio spectrum in the Sub-GHz Industrial, Scientific and Medical (ISM) bands with region-specific carrier frequencies and PHY parameter configurations. The LoRaWAN physical layer is based on Long Range (LoRa ${ }^{T M}$ ), a proprietary Chirp Spread Spectrum (CSS) modulation technique developed by Semtech, robust to multipath fading, Doppler shift and narrowband interference. Range and energy consumption of end devices depend on four parameters at the physical layer: (i) the channel bandwidth (BW), that defines the amplitude in the frequency domain of the used channel; (ii) the Spreading Factor (SF), that tells "how much" the reference signal is spread in time (the higher the SF, the longer the transmission range but also the lower the transmission rate); (iii) the Coding Rate (CR), that defines the redundancy of Forward Error Correction (FEC) optionally added to the LoRa messages; (iv) the transmission power $\left(P_{t x}\right)$.

The MAC level defines three classes of end devices: Class A devices transmit in the uplink using by standard a simple random ALOHA-based access protocol and can receive traffic in the downlink only after an uplink transmission. Class B devices can wake up periodically to receive scheduled downlink data traffic. Class C devices listen continuously and are typically mainspowered. Class A devices are, at the moment of writing, the ones with the highest diffusion in the market. To limit interference in the ISM band, Class A devices are mandated in Europe to operate with a duty cycle below $1 \%$ if running ALOHA access protocol, or, alternatively, adopting a Listen Before Talk approach with no limitations on the duty cycle.

\section{Problem Statement and Formulation}

We consider a single-gateway LoRaWAN servicing a set of end devices generating messages according to a Poisson process with rate $\lambda$. Each end device uses a specific SF in transmission out of the available six $(M=6)$. Two or more uplink transmissions can collide only if performed with the same SF. The set of end devices includes devices running plain ALOHA access scheme and devices accessing the channel via a Listen Before Talk approach based on an unslotted CSMA/CA protocol similar to the one used in the IEEE 802.15.4 standard.

According to this protocol, a device backs off transmission for a random number of backoff slots in the range $\left[0,2^{B E}-1\right]$, being $B E$ the backoff exponent that is initialized to $m_{\min }$. When the backoff expires, the device senses the availability of the channel through energy detection or other Clear Channel Assessment (CCA) techniques. If the channel is perceived as busy, the $B E$ is increased by 1 up to a maximum value $\left(m_{\max }\right)$ and the device backs off again for a period randomly generated with the new value of $B E$. This process is repeated until the number of failed CCAs exceeds the parameter $m$. In that case, the message is discarded. If the channel is clear, the device transmits the message.

Let $L_{l}$ be the airtime in ms of a message transmitted with SF $l$ (we assume fixed message size for all the end devices), and $N_{C, l}$ and $N_{A, l}$ the number of CSMA/CA and ALOHA end devices using SF $l$, respectively. We also denote with $t_{b}, t_{C C A}$ and $t_{T A}$ the duration of a backoff slot, of a CCA, and the turnaround time from the listening mode to the transmitting mode. For simplicity, we assume that $t_{C C A} \approx t_{T A} \approx t_{b} / 2$. We also assume that the CCA is based on pure energy detection, which means that the channel is perceived as busy if one message is being transmitted at CCA time, regardless of the used SF.

We rely on the Markov chain shown in Fig. 1 to model the backoff, sensing and transmitting states of the CSMA/CA devices. This approach has been widely used in the literature [12] - [15], so we give here the minimum set of equations needed to model the inner state behavior of the CSMA/CA devices, without giving a complete derivation of them.

A state is the tuple $(i, j)$, with $i$ the backoff stage and $j$ the backoff counter ranging from 0 to $W_{i}=2^{B E_{i}}-1$, with $B E_{i}$ the backoff exponent corresponding to the backoff stage $i$. In the states with $j=0$, CCA is performed. The probability that a device finds the channel busy when it performs a CCA is $\alpha$. If the number of end devices is high (as typically happens on LoRaWAN), we can safely assume that this probability is the same for all the CSMA/CA devices irrespective of their SF.

The states $(-1, j)$ represent the transmission of a message, with $0 \leq j<L_{l}^{\prime}$, and $L_{l}^{\prime}$ the duration in slots of a message transmitted using SF $l$ including overhead and payload. The traffic generation of the devices is modeled with a message generation probability in idle state $q_{l}$. We also include in the model the probabilities of having a message ready to be transmitted after a channel access failure $q_{c f, l}$ and after a transmission attempt $q_{t a, l}$. The expressions of these probabilities are derived afterward.

The probability $\tau_{l}$ that a device attempts a CCA in a randomly chosen time slot can be derived as in [15]:

$$
\tau_{l}=\left(\frac{1-\alpha^{m+1}}{1-\alpha}\right) p(0,0),
$$

where $p(0,0)$ is the steady state probability of the state $(0,0)$. The expression for $p(0,0)$ is given in Eq. (2). In that expression, $\hat{m}=m_{\max }-m_{\min }$. 


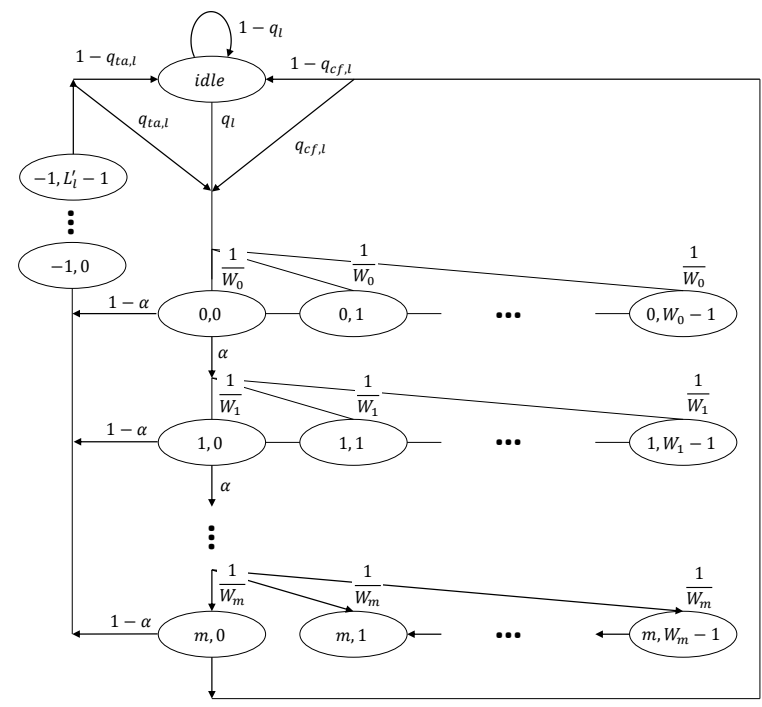

Fig. 1: Markov chain model of the unslotted CSMA/CA devices.

A channel can be found busy upon CCA because occupied by ALOHA and/or CSMA/CA transmissions. The corresponding probability, $\alpha$, can be written as

$$
\alpha=P\left(\mathcal{B}_{\mathcal{A}} \cup \mathcal{B}_{\mathcal{C}}\right)=P\left(\mathcal{B}_{\mathcal{A}}\right)+P\left(\mathcal{B}_{\mathcal{A}}^{C} \cap \mathcal{B}_{\mathcal{C}}\right),
$$

where $\mathcal{B}_{\mathcal{A}}$ indicates the event that channel is found busy because of an ALOHA transmission and $\mathcal{B}_{\mathcal{C}}$ indicates the event that the channel is busy because of a CSMA/CA transmission.

The term $P\left(\mathcal{B}_{\mathcal{A}}\right)$ is

$$
P\left(\mathcal{B}_{\mathcal{A}}\right)=1-\prod_{l=1}^{M} e^{-\lambda N_{A, l}\left(L_{l}+t_{C C A}\right)},
$$

i.e., the probability that any ALOHA device starts its transmission in the $L_{l}+t_{C C A}$ seconds before the beginning of the CCA.

On the other hand, the term $P\left(\mathcal{B}_{\mathcal{A}}^{C} \cap \mathcal{B}_{\mathcal{C}}\right)$ can be expressed as

$$
\begin{aligned}
P & \left(\mathcal{B}_{\mathcal{A}}^{C} \cap \mathcal{B}_{\mathcal{C}}\right)=P\left(\mathcal{B}_{\mathcal{A}}^{C} \bigcap\left(\bigcup_{l=1}^{M} \mathcal{B}_{\mathcal{C}, l}\right)\right) \\
& =\sum_{l=1}^{M} P\left(\mathcal{B}_{\mathcal{A}}^{C} \bigcap \mathcal{B}_{\mathcal{C}, l} \bigcap_{m=1}^{l-1} \mathcal{B}_{\mathcal{C}, m}^{C}\right) \\
& =\sum_{l=1}^{M}\left[P\left(\mathcal{B}_{\mathcal{A}}^{C} \mid \mathcal{B}_{\mathcal{C}, l} \bigcap_{m=1}^{l-1} \mathcal{B}_{\mathcal{C}, m}^{C}\right) P\left(\mathcal{B}_{\mathcal{C}, l} \bigcap_{m=1}^{l-1} \mathcal{B}_{\mathcal{C}, m}^{C}\right)\right] .
\end{aligned}
$$

with $\mathcal{B}_{\mathcal{C}, l}$ the event that the CCA fails because there is transmitting a CSMA/CA device with SF $l$. Additionally, $\bigcap_{m=1}^{l-1} \mathcal{B}_{\mathcal{C}, m}^{C}=\Omega$ when $j=1$.
Now we derive the two probabilities on the previous expression. First, we can rewrite

$$
P\left(\mathcal{B}_{\mathcal{C}, l} \bigcap_{m=1}^{l-1} \mathcal{B}_{\mathcal{C}, m}^{C}\right)=P\left(\bigcap_{m=1}^{l-1} \mathcal{B}_{\mathcal{C}, m}^{C} \mid \mathcal{B}_{\mathcal{C}, l}\right) P\left(\mathcal{B}_{\mathcal{C}, l}\right) .
$$

The term $P\left(\mathcal{B}_{\mathcal{C}, l}\right)$ is related with the probability that at least one device using SF $l$ has accessed the channel and found it free multiplied with the duration of the message it transmits (this term corresponds to the probability to sense busy in a CSMA/CA network without ALOHA devices [16]). Therefore,

$$
P\left(\mathcal{B}_{\mathcal{C}, l}\right)=\left(1-\left(1-\tau_{l}\right)^{N_{C, l}}\right)(1-\alpha) L_{l}^{\prime} .
$$

Leveraging the commutativity of set union, we can assume that $l=1$ corresponds to the SF for which the messages last longer and that the different SFs are ordered in descending order of its duration (i.e $L_{i}<L_{j}$ for $j<i$ ). Additionally, if the CCA fails because of the transmission of some CSMA/CA device using SF $l$, this CSMA/CA device must have found the channel empty on its CCA. This implies that no other device can be transmitting before this CCA. Consequently, the only case where a CSMA/CA device using SF $m<l$ could also transmit and occupy the channel is if it performs its CCA at the same time as the CSMA/CA device using SF $l$. Therefore

$$
P\left(\bigcap_{m=1}^{l-1} \mathcal{B}_{\mathcal{C}, m}^{C} \mid \mathcal{B}_{\mathcal{C}, l}\right)=\prod_{m=1}^{l-1}\left(1-\tau_{m}\right)^{N_{C, m}},
$$

i.e., the probability that no CSMA/CA device using SF $m<l$ performs a CCA at the same time as the device using SF $l$ that has occupy the channel.

The same assumption $\left(L_{i}<L_{j}\right.$ for $\left.j<i\right)$ also allows simplifying the first probability in Eq. (5). The event $\mathcal{B}_{\mathcal{C}, l}$ implies that no other device is transmitting when the CSMA/CA device causing the failed CCA performs its, and the event $\bigcap_{m=1}^{l-1} \mathcal{B}_{\mathcal{C}, m}^{C}$ implies that no other CSMA/CA device with SF $m<l$ performs the CCA at the same time as the CSMA/CA device causing the event $\mathcal{B}_{\mathcal{C}, l}$. From the point of view of ALOHA transmissions, the only meaningful event is $\mathcal{B}_{\mathcal{C}, l}$ as it forces that there cannot be an ALOHA transmission prior to the CCA of the CSMA/CA device occupying the channel. Therefore

$$
P\left(\mathcal{B}_{\mathcal{A}}^{C} \mid \mathcal{B}_{\mathcal{C}, l} \bigcap_{m=1}^{l-1} \mathcal{B}_{\mathcal{C}, m}^{C}\right)=P\left(\mathcal{B}_{\mathcal{A}}^{C} \mid \mathcal{B}_{\mathcal{C}, l}\right)
$$

Given that ALOHA transmissions are independent amongst them, Eq. (9) can be rewritten as

$$
P\left(\mathcal{B}_{\mathcal{A}}^{C} \mid \mathcal{B}_{\mathcal{C}, l}\right)=\prod_{m=1}^{M} P\left(\mathcal{B}_{\mathcal{A}, m}^{C} \mid \mathcal{B}_{\mathcal{C}, l}\right)
$$

with $\mathcal{B}_{\mathcal{A}, m}$ the event that the CCA fails because of an ALOHA transmission using SF $m$. As event $\mathcal{B}_{\mathcal{C}, l}$ 


$$
p_{l}(0,0)=\left\{\begin{array}{l}
{\left[\frac{1}{2}\left(\frac{1-(2 \alpha)^{m+1}}{1-2 \alpha} W_{0}+\frac{1-\alpha^{m+1}}{1-\alpha}\right)+L_{l}^{\prime}\left(1-\alpha^{m+1}\right)+\frac{1-q_{c f, l}}{q_{l}} \alpha^{m+1}+\frac{1-q_{t a, l}}{q_{l}}\left(1-\alpha^{m+1}\right)\right]^{-1}, \text { if } m<\hat{m}} \\
{\left[\frac{1}{2}\left(\frac{1-(2 \alpha)^{\hat{m}+1}}{1-2 \alpha} W_{0}+\frac{1-\alpha^{\hat{m}+1}}{1-\alpha}+\left(2^{m_{b}+1}+1\right) \alpha^{\hat{m}+1} \frac{1-\alpha^{m-\hat{m}}}{1-\alpha}\right)+L_{l}^{\prime}\left(1-\alpha^{m+1}\right)+\frac{1-q_{c f, l}}{q_{l}} \alpha^{m+1}\right.} \\
\left.+\frac{1-q_{t a, l}}{q_{l}}\left(1-\alpha^{m+1}\right)\right]^{-1}, \text { otherwise }
\end{array}\right.
$$

ensures that there is no ALOHA transmissions before the CCA of the transmission that has occupied the channel, the term $P\left(\mathcal{B}_{\mathcal{A}, m}^{C} \mid \mathcal{B}_{\mathcal{C}, l}\right)$ has to consider only transmissions after that CCA. In order to compute this probability, we consider two cases. If $L_{m}>L_{l}$, $P\left(\mathcal{B}_{\mathcal{A}, m}^{C} \mid \mathcal{B}_{\mathcal{C}, l}\right)$ corresponds to the probability that no ALOHA transmission using SF $m$ starts from the beginning of the turn-around time of the CSMA/CA transmission using SF $l$ to the end of the CCA. As all the transmitting states in the Markov chain of Fig. 1 have the same probability, we can assume that the beginning of that CSMA/CA transmission is uniformly distributed in $\left[-L_{l}, t_{C C A}\right]$ (considering that the CSMA/CA device that finds the channel busy starts its CCA at time instant $0)$. With this, we have

$$
\begin{gathered}
P\left(\mathcal{B}_{\mathcal{A}, m}^{C} \mid \mathcal{B}_{\mathcal{C}, l}\right)=\int_{-L_{l}}^{t_{C C A}} \frac{e^{-\lambda N_{A, m}\left(t_{C C A}-t+t_{T A}\right)}}{L_{l}+t_{C C A}} d t \\
=\frac{e^{\lambda N_{A, m} t_{T A}}\left(1-e^{-\lambda N_{A, m}\left(L_{l}+t_{C C A}\right)}\right)}{\lambda N_{A, m}\left(L_{l}+t_{C C A}\right)} .
\end{gathered}
$$

The integrand of the previous equation indicates that if the CSMA/CA transmission with SF $l$ that has occupied the channel has begun at time instant $t \in$ $\left[-L_{l}, t_{C C A}\right]$, there cannot be any ALOHA transmission with SF $m$ in the time period $\left[t-t_{T A}, t_{C C A}\right]$.

If $L_{m} \leq L_{l}$, we have to consider two cases depending on the instant at which the transmission that has produced the event $\mathcal{B}_{\mathcal{C}, l}$ starts. If it has begun at $t \in\left[-L_{l},-L_{m}+t_{T A}\right]$, event $\mathcal{B}_{\mathcal{A}, m}^{C} \mid \mathcal{B}_{\mathcal{C}, l}$ implies that there has not been any ALOHA transmission with SF $m$ in $\left[-L_{m}, t_{C C A}\right]$. On the contrary, if it has begun at $t \in\left[-L_{m}+t_{T A}, t_{T A}\right]$, then there cannot have been any ALOHA transmission in $\left[t-t_{T A}, t_{C C A}\right]$. Therefore,

$$
\begin{gathered}
P\left(\mathcal{B}_{\mathcal{A}, m}^{C} \mid \mathcal{B}_{\mathcal{C}, l}\right)=\int_{-L_{l}}^{-L_{m}+t_{T A}} \frac{e^{-\lambda N_{A, m}\left(L_{m}+t_{C C A}\right)}}{L_{l}+t_{C C A}} d t \\
+\int_{-L_{m}+t_{T A}}^{t_{T A}} \frac{e^{-\lambda N_{A, m}\left(t_{C C A}-t t_{T A}\right)}}{L_{l}+t_{C C A}} d t \\
=\frac{\left(L_{l}-L_{m}+t_{T A}\right) e^{-\lambda N_{A, m}\left(L_{m}+t_{C C A}\right)}}{L_{l}+t_{C C A}} \\
+\frac{e^{-\lambda N_{A, m} t_{T A}}-e^{-\lambda N_{A, m}\left(L_{m}+t_{C C A}\right)}}{\lambda N_{A, m}\left(L_{l}+t_{C C A}\right)}
\end{gathered}
$$

Note that $P\left(\mathcal{B}_{\mathcal{A}, m}^{C} \mid \mathcal{B}_{\mathcal{C}, l}\right)=1$ in eqs. (11) and (12) if $N_{A, m}$ is zero.

Eqs. (1) (one for each SF) and (3) form a system of $M+1$ coupled nonlinear equations with variables $\tau_{l}$ and $\alpha$ that can be solved numerically to obtain the point of operation of the network. From these variables different performance metrics can be obtained. First, the probability that a message transmitted by an CSMA/CA device using SF $l$ collides is

$$
\begin{aligned}
P_{c, l, C} & =P\left(\mathcal{C}_{l, \mathcal{A}}\right)+P\left(\mathcal{C}_{l, \mathcal{C}}\right)-P\left(\mathcal{C}_{l, \mathcal{A}} \cap \mathcal{C}_{l, \mathcal{C}}\right) \\
& =P\left(\mathcal{C}_{l, \mathcal{A}}\right)+P\left(\mathcal{C}_{l, \mathcal{C}}\right)\left(1-P\left(\mathcal{C}_{l, \mathcal{A}}\right)\right) .
\end{aligned}
$$

In this expression, $\mathcal{C}_{l, \mathcal{A}}$ (or $\mathcal{C}_{l, \mathcal{C}}$ ) corresponds to the event that the CSMA/CA device using SF $l$ collides with an ALOHA (or CSMA/CA) device using its same SF. Note that the last step in Eq. (13) can be done as the probability of colliding with a CSMA/CA device is independent of the probability of colliding with an ALOHA device. The reason of this independence is that the event $\mathcal{C}_{l, \mathcal{C}}$ does not add any information to the event $\mathcal{C}_{l, \mathcal{A}}$ as the only possibility for a CSMA/CA device to collide with another CSMA/CA device is when both begin its transmission simultaneously.

The term $P\left(\mathcal{C}_{l, \mathcal{A}}\right)$ is

$$
P\left(\mathcal{C}_{l, \mathcal{A}}\right)=1-e^{-\lambda N_{A, l}\left(L_{l}+t_{T A}\right)},
$$

which corresponds to the probability that there is at least one transmission of an ALOHA device using SF $l$ during the transmission of the CSMA/CA device. Similarly, $P\left(\mathcal{C}_{l, \mathcal{C}}\right)$ is the probability that any other CSMA/CA device performs the CCA at the same time,

$$
P\left(\mathcal{C}_{l, \mathcal{C}}\right)=1-\left(1-\tau_{l}\right)^{N_{C, l}-1} .
$$

With this, the DER of a CSMA/CA device using SF $l$ corresponds to the probability of a successful transmission

$$
D E R_{l, C}=\left(1-P_{c, l, C}\right)\left(1-\alpha^{m+1}\right),
$$

i.e., the probability that it finds the channel idle in any of the CCA attempts times the probability that the message does not collide given that it is transmitted.

We derive now the expressions for the average delay experienced by a CSMA/CA message. We distinguish between two different cases: (i) when the channel has been found idle in any of the $m+1$ allowed CCAs and the message has been transmitted and (ii) when the message is discarded because of a channel access failure (i.e., the channel has been found busy in the $m+1$ CCAs). To compute these metrics, we consider only the interval from the time the message is ready to be transmitted (i.e., we exclude any queuing time).

In the first case, the average delay is

$$
\mathrm{E}\left[T_{t a, l}\right]=L_{l}+\mathrm{E}\left[T_{b}\right],
$$


being $T_{b}$ the random time that a device spends in backoff or sensing states during the CSMA/CA mechanism. The expected value of $T_{b}$ is

$$
\mathrm{E}\left[T_{b}\right]=\sum_{i=0}^{m} P\left(\mathcal{D}_{i}\right) \mathrm{E}\left[T_{b, i}\right],
$$

where $P\left(\mathcal{D}_{i}\right)$ is the probability of finding the channel idle at the $i+1$ th CCA attempt, given that the channel has been found busy in the preceding $i$ attempts and the message has not been discarded due to a channel access failure; and $\mathrm{E}\left[T_{b, i}\right]$ is the expected time a device spends in backoff or sensing states given the event $\mathcal{D}_{i}$. $P\left(\mathcal{D}_{i}\right)$ can be calculated as

$$
P\left(\mathcal{D}_{i}\right)=\frac{\alpha_{l}^{i}}{\sum_{k=0}^{m} \alpha_{l}^{k}} \alpha_{l}^{i}=\frac{1-\alpha_{l}}{1-\alpha_{l}^{m+1}},
$$

while

$$
\begin{aligned}
\mathrm{E}\left[T_{b, i}\right] & =(i+1) t_{C C A}+\sum_{k=0}^{i} \mathrm{E}\left[B_{k}\right] t_{b} \\
& =(i+1) t_{C C A}+\sum_{k=0}^{i} t_{b} \frac{W_{k}-1}{2},
\end{aligned}
$$

with $B_{k}=\mathcal{U}\left(0, W_{k}\right)$ a discrete uniform random variable indicating the backoff outcome at backoff stage $k$.

The delay suffered by a message when it is discarded due to a channel access failure is

$$
\mathrm{E}\left[T_{c f, l}\right]=\sum_{k=0}^{m} t_{b} \frac{W_{k}-1}{2}
$$

which is independent of the SF used by the CSMA/CA device.

Finally, the probability of having a message ready to be transmitted in idle state is $q_{l}=1-e^{-\lambda_{l} t_{b}}$ (i.e., the probability of a message arrival in one slot). The probabilities of having a message ready to be transmitted after a transmission attempt and after a channel access failure can be approximated with the busy server probability of a non saturated system obtaining $q_{t a, l}=\lambda \mathrm{E}\left[T_{t a, l}\right]$ and $q_{c f, l}=\lambda \mathrm{E}\left[T_{c f, l}\right]$.

The DER for an ALOHA device using SF $l$ is its probability of having a successful transmission

$$
\begin{aligned}
& D E R_{A, l}=1-P\left(\mathcal{A}_{\mathcal{A}, l} \cup \mathcal{A}_{\mathcal{C}, l}\right) \\
& \quad=1-P\left(\mathcal{A}_{\mathcal{A}, l}\right)-P\left(\mathcal{A}_{\mathcal{A}, l}^{C} \mid \mathcal{A}_{\mathcal{C}, l}\right) P\left(\mathcal{A}_{\mathcal{C}, l}\right),
\end{aligned}
$$

where $\mathcal{A}_{\mathcal{A}, l}$ indicates the event that the ALOHA device collides with another ALOHA device using its same SF and $\mathcal{A}_{\mathcal{C}, l}$ indicates the event that the ALOHA device collides with a CSMA/CA device.

The term $P\left(\mathcal{A}_{\mathcal{A}, l}\right)$ is the probability that there is at least one transmission of another ALOHA device using the same $\mathrm{SF}$ in the timespan $2 L_{l}$, which is

$$
P\left(\mathcal{A}_{\mathcal{A}, l}\right)=1-e^{-2 \lambda\left(N_{A, l}-1\right) L_{l}}
$$

Likewise, $P\left(\mathcal{A}_{\mathcal{C}, l}\right)$ is the probability that at least one CSMA/CA device with the same SF has begun a transmission in the $L_{l}+t_{T A}$ seconds before the transmission attempt of the ALOHA device,

$$
P\left(\mathcal{A}_{\mathcal{C}, l}\right)=\left(1-\left(1-\tau_{l}\right)^{N_{C, l}}\right)(1-\alpha)\left(L_{l}^{\prime}+\frac{t_{T A}}{t_{b}}\right)
$$

The conditional probability $P\left(\mathcal{A}_{\mathcal{A}, l}^{C} \mid \mathcal{A}_{\mathcal{C}, l}\right)$ corresponds to the probability there is not another ALOHA transmission from the beginning of the turn-around time of the CSMA/CA transmission causing the collision to the end of the collided ALOHA transmission. We can assume that the beginning of the colliding CSMA/CA transmission is uniformly distributed in the time period $\left[-L_{l}, t_{T A}\right]$ (considering that the beginning of the collided ALOHA transmission occurs at time instant $0)$. With this, if the colliding CSMA/CA transmission has begun at a time instant $t \in\left[-L_{l},-L_{l}+t_{T A}\right]$, the event $\mathcal{A}_{\mathcal{A}, l}^{C} \mid \mathcal{A}_{\mathcal{C}, l}$ implies that there has not been another ALOHA transmission in the time period $\left[-L_{l}, L_{l}\right]$. Similarly, if the colliding CSMA/CA transmission has begun at time instant $t \in\left[-L_{l}+t_{T A}, t_{T A}\right]$, then there cannot have been another ALOHA transmission in the time period $\left[t-t_{T A}, L_{l}\right]$. Therefore,

$$
\begin{gathered}
P\left(\mathcal{A}_{\mathcal{A}, l}^{C} \mid \mathcal{A}_{\mathcal{C}, l}\right)=\int_{-L_{l}}^{-L_{l}+t_{T A}} \frac{e^{-2 \lambda\left(N_{A, l}-1\right) L_{l}}}{L_{l}+t_{T A}} d t \\
\quad+\int_{-L_{l}+t_{T A}}^{t_{T A}} e^{-\lambda\left(N_{A, l}-1\right)\left(L_{l}-t+t_{T A}\right)} d t \\
=\frac{t_{T A} \cdot e^{-2 \lambda\left(N_{A, l}-1\right) L_{l}}}{L_{l}+t_{T A}} \\
+\frac{e^{-\lambda\left(N_{A, l}-1\right) L_{l}}-e^{-2 \lambda\left(N_{A, l}-1\right) L_{l}}}{\lambda\left(N_{A, l}-1\right)\left(L_{l}+t_{T A}\right)} .
\end{gathered}
$$

\section{NumERiCAL RESUlts}

We consider a system with 300 end devices (50 devices per SF) served by a single gateway and different configurations of ALOHA and CSMA/CA devices. To validate the proposed model, we built up a system-level discrete event simulator written in $\mathrm{C}++$. The simulation results shown hereafter are obtained by averaging of ten simulation runs consisting each of $10^{7}$ frame transmissions (we do not represent the $95 \%$-confidence intervals as their relative size is well below 1\%).

The LoRA parameters used in the simulations are listed in Table I. The MAC parameters used in this section are $m_{\min }=m_{\max }=12$ and $m=4$. We have also set the duration of a backoff slot to $1.4 \mathrm{~ms}$, which corresponds to the time required to transmit the 8 bytes of the LoRaWAN physical layer preamble using the SF 7 with a bandwidth of $125 \mathrm{kHz}$. The election of large backoff exponents is due to the long duration of the messages transmitted using high SFs (for instance, the duration of a message transmitted with SF 12 is 1293 slots). If they were not that long, the probability 


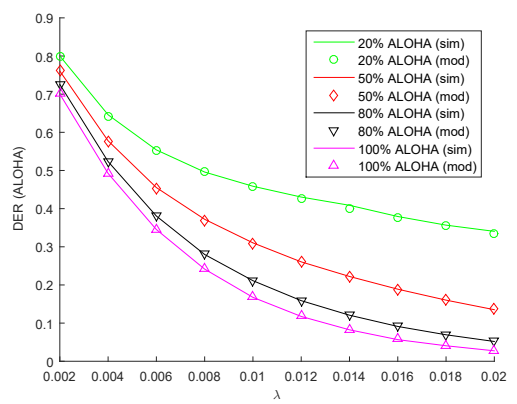

(a) $L=1810.43 \mathrm{~ms}(\mathrm{SF}=12)$

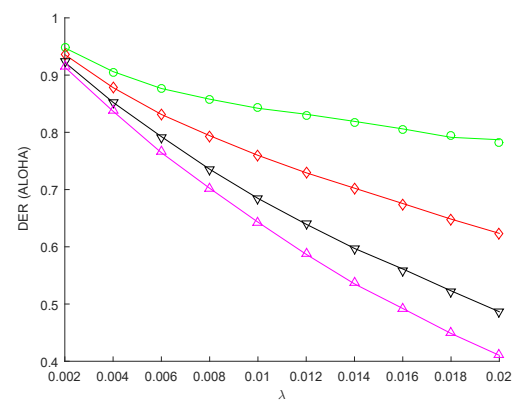

(b) $L=452.61 \mathrm{~ms}(\mathrm{SF}=10)$

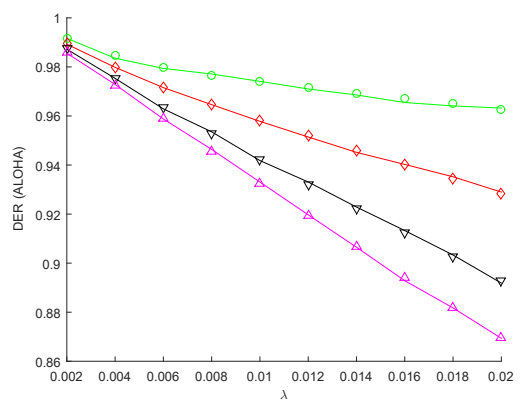

(c) $L=71.94 \mathrm{~ms}(\mathrm{SF}=7)$

Fig. 2: DER for CSMA/CA devices versus message generation rate

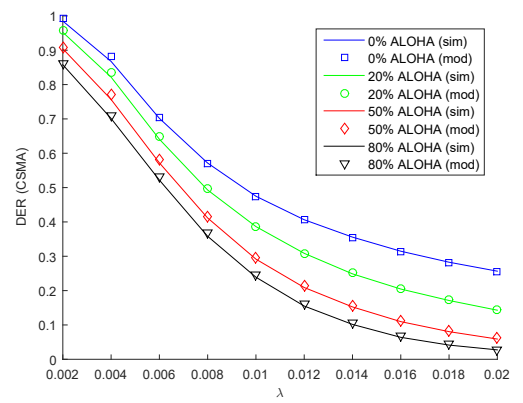

(a) $L=1810.43 \mathrm{~ms}(\mathrm{SF}=12)$

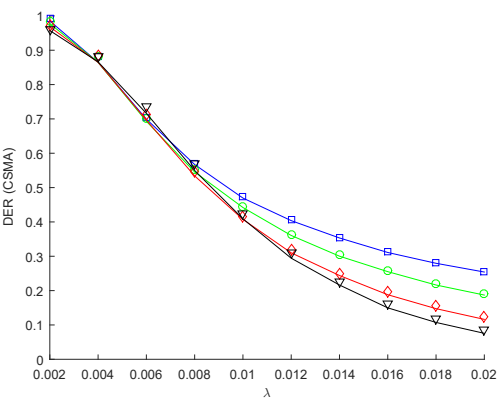

(b) $L=452.61 \mathrm{~ms}(\mathrm{SF}=10)$

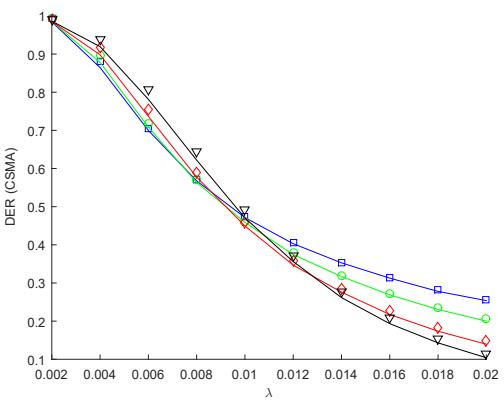

(c) $L=71.94 \mathrm{~ms}(\mathrm{SF}=7)$

Fig. 3: DER for CSMA/CA devices versus message generation rate

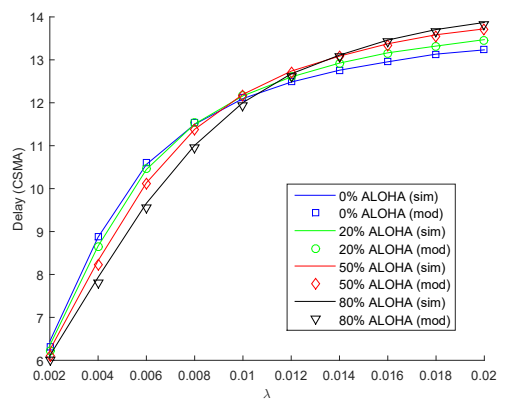

(a) $L=1810.43 \mathrm{~ms}(\mathrm{SF}=12)$

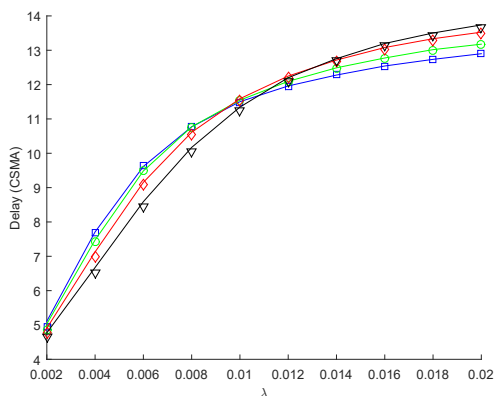

(b) $L=452.61 \mathrm{~ms}(\mathrm{SF}=10)$

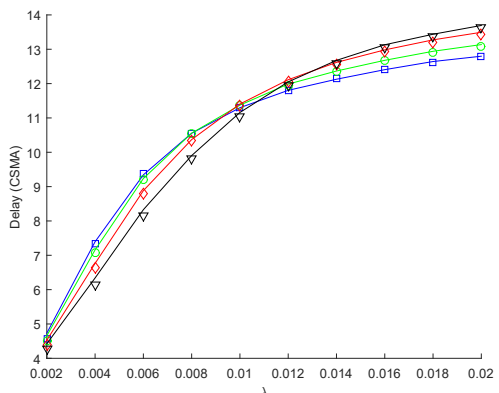

(c) $L=71.94 \mathrm{~ms}(\mathrm{SF}=7)$

Fig. 4: Average delay in seconds for CSMA/CA devices versus message generation rate

of finding the channel busy in successive CCAs given that it was busy in the first CCA would be high since the transmitted message(s) causing the first failed CCA may not has ended in the next CCA.

\section{TABLE I: LoRaWAN PHY Parameters}

\begin{tabular}{|c|c|c|c|c|c|c|}
\hline Application payload [byte] & \multicolumn{7}{|c|}{13} \\
\hline LoRaWAN header [byte] & \multicolumn{7}{|c|}{8} \\
\hline Coding rate & \multicolumn{7}{|c|}{125} \\
\hline Preamble [byte] & \multicolumn{7}{|c|}{10} & 11 & 12 \\
\hline Bandwidth [kHz] & 7 & 8 & 9 & 10 & 18 \\
\hline SF & 71.94 & 133.63 & 246.78 & 452.60 & 987.13 & 1810.43 \\
\hline Air Time [ms] & &
\end{tabular}

Fig. 2 shows the DER of ALOHA devices for different SFs, message generation rates and percentages of ALOHA and CSMA/CA devices. These graphs (as well as in those on Figs. 3 and 4) were obtained under uniform SF assignment to end devices, that is, with 50 devices using each SF. Therefore, the 20\% ALOHA line indicates that there are 10 ALOHA devices in each $\mathrm{SF}$ and 60 ALOHA devices in total. As can be seen, the inclusion of CSMA/CA devices in a LoRaWAN network impacts positively on the probability of having a successful transmission for ALOHA devices in all the cases, independently of the message generation rate and the specific SF used by the devices. The reason behind this behavior is evident: if there is an ALOHA transmission on the channel, CSMA/CA devices will detect it and will refrain from transmitting, thus decreasing the collision probability of ALOHA messages.

Similarly, Fig. 3 shows the DER of CSMA/CA 
devices for different SFs, message generation rates and percentages of ALOHA and CSMA/CA devices. As clear from the figure, similarly to what observed for ALOHA devices, the probability of a successful transmission for CSMA/CA devices using high values of SF increases as the fraction of ALOHA devices decreases. On the contrary, for low values of SF, it is better to have a higher percentage of ALOHA devices when $\lambda$ is also low. The reason behind this is the following: the prevailing source of error for CSMA/CA devices with low values of SF is a channel access failure, whose probability for low $\lambda$ is greater when the number of CSMA/CA devices in the network is large. On the other hand, for high $\lambda$ this probability is greater when the number of ALOHA devices is large. For low values of $\lambda$, CSMA/CA devices have higher successful probabilities than ALOHA devices, while for large values of $\lambda$ ALOHA devices tend to obtain better results. This behavior is due to the fact that for high $\lambda$, the main error source for CSMA/CA devices is channel access failures. This kind of errors refrains CSMA/CA devices from transmitting, which lowers the collision probability for ALOHA devices. Finally, the probability of a successful transmission amongst the different SF is more similar for CSMA/CA devices than for ALOHA devices, which is due to the fact that the probability of finding the channel busy do not depend of the SF.

Fig. 3 shows the average delay suffered by a CSMA/CA message from the moment it initiates the backoff process until it is received (wrongly or not) or discarded due to a channel access failure. The depicted results correspond to

$$
T_{l}=\alpha^{m+1} \mathrm{E}\left[T_{t a, l}\right]+\left(1-\alpha^{m+1}\right) \mathrm{E}\left[T_{c f, l}\right] .
$$

In this case, the average delay is similar for all the SF, as this delay depends mainly on the term $\alpha^{m+1} \mathrm{E}\left[T_{t a, l}\right]$, which is the same for all the SFs.

As a final remark, it is worth mentioning that our model fits closely the results obtained with simulations in all the cases, demonstrating its accurateness and utility to obtain performance results with a low computational cost.

\section{COnCluding Remarks}

We have addressed the performance evaluation of LoRaWAN Class A end devices by introducing a theoretical framework for the modeling of the Data Extraction Rate and the average transmission delay in the uplink segment. The proposed model was used to evaluate the coexistence in the same network of two different MAC schemes: plain ALOHA and Listen Before Talk based on CSMA/CA through energy detection. The numerical results of the model validated against simulation demonstrate the model is indeed successful in capturing the performance of LoRaWAN networks.
To the best of our knowledge, ours is the first work assessing the performance of "mixed" MAC situations.

\section{ACKNOWLEDGMENT}

J. Ortin was supported by the Spanish Government through grant CAS17/00624 from the mobility program of the Ministerio de Educación, Cultura y Deporte, grant TEC2014-52969-R from the Ministerio de Ciencia e Innovación, Universidad de Zaragoza through grant UZ2018-TEC-04, Gobierno de Aragón and FEDER "Construyendo Europa desde Aragón", and Centro Universitario de la Defensa through project CUD2017-18.

\section{REFERENCES}

[1] A. Rico-Alvarino et al., "An overview of 3GPP enhancements on machine to machine communications," IEEE Communications Magazine, vol. 54, no. 6, pp. 14-21, June 2016.

[2] "LoRa alliance technology," https://www.lora-alliance.org/.

[3] J. Petajajarvi et al., "Performance of a low-power wide-area network based on LoRa technology: Doppler robustness, scalability, and coverage," International Journal of Distributed Sensor Networks, vol. 13, no. 3, pp. 55-59, March 2017.

[4] K. Mikhaylov et al., "On LoRaWAN scalability: Empirical evaluation of susceptibility to inter-network interference," in 2017 European Conference on Networks and Communications (EuCNC), June 2017, pp. 1-6.

[5] - "D2D communications in LoRaWAN low power wide area network: From idea to empirical validation," in 2017 IEEE International Conference on Communications Workshops (ICC Workshops), May 2017, pp. 737-742.

[6] A. Pop et al., "Does bidirectional traffic do more harm than good in LoRaWAN based LPWA networks?" CoRR, vol. abs/1704.04174, 2017. [Online]. Available: http://arxiv.org/abs/1704.04174

[7] J. Haxhibeqiri et al., "LoRa scalability: A simulation model based on interference measurements," Sensors, vol. 17, no. 6, 2017. [Online]. Available: http://www.mdpi.com/1424$8220 / 17 / 6 / 1193$

[8] F. Adelantado et al., "Understanding the limits of LoRaWAN," IEEE Communications Magazine, vol. 55, no. 9, pp. 34-40, 2017.

[9] F. Delobel et al., "Analysis of the delay of confirmed downlink frames in class B of LoRaWAN," in 2017 IEEE 85th Vehicular Technology Conference (VTC Spring), June 2017, pp. 1-6.

[10] O. Georgiou and U. Raza, "Low power wide area network analysis: Can LoRa scale?" IEEE Wireless Communications Letters, vol. 6, no. 2, pp. 162-165, April 2017.

[11] D. Zucchetto and A. Zanella, "Uncoordinated access schemes for the IoT: Approaches, regulations, and performance," IEEE Communications Magazine, vol. 55, no. 9, pp. 48-54, 2017.

[12] S. Pollin et al., "Performance analysis of slotted carrier sense IEEE 802.15.4 medium access layer," IEEE Transactions on Wireless Communications, vol. 7, no. 9, pp. 3359-3371, September 2008.

[13] P. Park et al., "A generalized Markov chain model for effective analysis of slotted IEEE 802.15.4," in 2009 IEEE 6th International Conference on Mobile Adhoc and Sensor Systems, Oct 2009, pp. 130-139.

[14] K. Govindan et al., "Modeling and analysis of non beacon mode for low-rate WPAN," in 2015 12th Annual IEEE Consumer Communications and Networking Conference (CCNC), Jan 2015, pp. 549-555.

[15] P. D. Marco et al., "Analytical modeling of multi-hop IEEE 802.15.4 networks," IEEE Transactions on Vehicular Technology, vol. 61, no. 7, pp. 3191-3208, Sept 2012.

[16] S. Pollin et al., "Performance analysis of slotted carrier sense IEEE 802.15.4 medium access layer," in IEEE Globecom 2006, Nov. 2006, pp. 1-6. 